International Journal of Engineering \& Technology, $7(4.10)(2018) 460-463$
International Journal of Engineering \& Technology
SPC
Website: www.sciencepubco.com/index.php/IJET
Research paper

\title{
k-Zumkeller Labeling of Graphs
}

\author{
B.J. Balamurugan ${ }^{1}$, K. Thirusangu ${ }^{2}$, D.G. Thomas ${ }^{3}$ and B.J. Murali ${ }^{4}$ \\ ${ }^{I}$ School of Advanced Sciences, VIT University \\ Chennai Campus, Chennai - 600 127, India. \\ ${ }^{2}$ Department of Mathematics, SIVET College \\ Gowrivakkam, Chennai - 600 073, India. \\ ${ }^{3}$ Department of Mathematics, Madras Christian College \\ Tambaram, Chennai - 600 059, India. \\ ${ }^{4}$ Research Scholar, Research and Development Centre \\ Bharathiar University, Coimbatore - 641 046, India. \\ *Corresponding authorE-mail: balamurugan.bj@vit.ac.in
}

\begin{abstract}
In this paper, we mainly focus on to prove that the graphs, viz., (i)paths, (ii) comb graphs, (iii) cycles, (iv) ladder graphs and (v) $P_{n} \times P_{n}$ graphs are k-Zumkeller graphs.
\end{abstract}

Keywords: Graphs; labeling; Zumkeller numbers; $k$-Zumkeller labeling.

\section{Introduction}

The classes of labeled graphs can be identified through graph labeling, an interesting and potential research area of discrete mathematics. The labeled graphs finds many interesting applications in science, engineering and technology and we refer [6] for the same. The basic idea of the graph labeling is found in [11]. The collection of various graph labeling can be found in [7]. We refer the text book written by Harary [8] for notations and terminology in graph theory. There is a strong link between number theory and graph theory, the two interesting topics of mathematics. The graphs labeled with Zumkeller numbers [13], a recent development in graph labeling, are potential area of research. In [1, 2, 3, 4, 9], we have investigated two different graph labeling viz., (i) Zumkeller labeling and (ii) Strongly multiplicative Zumkeller labeling. In [5], we introduced the k-Zumkeller graphs and proved that the twig graphs are 4-Zumkeller graphs. In this paper, we further prove that the following interesting graphs, viz., (i) paths, (ii) cycles, (iii) comb graphs, (iv) ladder graphs and (v) $\mathrm{P}_{\mathrm{n}} \times \mathrm{P}_{\mathrm{n}}$ graphs are k-Zumkeller graphs.

\section{Zumkeller Numbers}

In this section, we see the definition of Zumkeller numbers. In the year 2003, Zumkeller had generalized the concept of perfect numbers [12] and published a sequence of positive integers known as Zumkeller numbers in A083207 integer (Sloane's) sequence. A Zumkeller number [13] $\mathrm{n}$ can be defined as follows.

\section{Definition 2.1. [13]}

Let $\mathrm{n}$ be a positive integer. The integer $\mathrm{n}$ is called a Zumkeller number if the two disjoint sets of positive factors of $n$ should have the same total. That is, the sum of the positive factors of the two disjoint sets is equal. The disjoint partition is called as Zumkeller partition.

For example, the positive integer 30 is a Zumkeller number because its positive factors are partitioned into $A=\{6,30\}$ and $B=$ $\{1,2,3,5,10,15\}$ such that sum of the elements of $\mathrm{A}$ is equal to the sum of the elements of $B$.

For properties of Zumkeller Numbers, we refer [13].

\section{Main Results}

This section introduces the concept of the k-Zumkeller labeling of a graph introduced by us in [5] and we show that certain graphs are $\mathrm{k}-Z$ Zumkeller graphs.

Definition 3.1. [5]

Let $\mathrm{G}=(\mathrm{V}, \mathrm{E})$ be a simple graph. A $1-1$ function $\mathrm{f}: \mathrm{V} \rightarrow \mathrm{N}$ is called a k-Zumkeller labeling of the graph $\mathrm{G}$ if the induced edge function $\mathrm{f}^{*}: \mathrm{E} \rightarrow \mathrm{N}$ defined by $\mathrm{f}^{*}(\mathrm{xy})=\mathrm{f}(\mathrm{x}) \mathrm{f}(\mathrm{y}), \mathrm{x} \in \mathrm{V}, \mathrm{y} \in \mathrm{V}$, $x y \in E$ satisfies the following two conditions:

(i) $f^{*}(x y)$ is a Zumkeller number for all $x y \in E$.

(ii) The number of different Zumkeller numbers used to label the edges of $\mathrm{G}$ is $\mathrm{k}$.

Definition 3.2. [5]

Let $\mathrm{G}=(\mathrm{V}, \mathrm{E})$ be a simple graph. If the graph $\mathrm{G}$ assumes a $\mathrm{k}$ Zumkeller labeling then it is known as a k-Zumkeller graph.

For example, a 4-Zumkeller labeling pattern of a graph is given in Figure 1. 


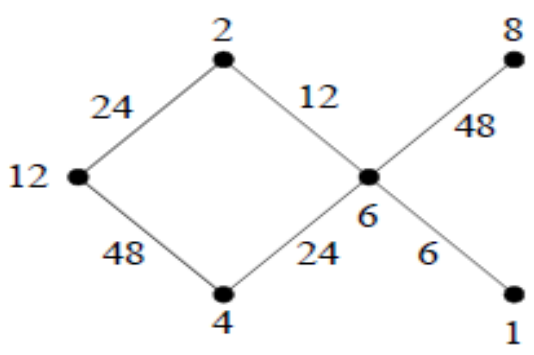

Fig1: A 4-Zumkeller graph

\section{Lemma 3.1.}

A sub graph of a k-Zumkeller graph need not be a k-Zumkeller graph.

\section{Lemma 3.2.}

The path $P_{n}$ with $n$ vertices is a 2-Zumkeller graph when $n \equiv 1$ $(\bmod 2)$ and $\mathrm{n} \geq 3$.

\section{Proof.}

Let $\mathrm{V}=\left\{\mathrm{v}_{\mathrm{i}} \mid 1 \leq \mathrm{i} \leq \mathrm{n}\right\}$ be the vertex set and $\mathrm{E}=\left\{\mathrm{e}_{\mathrm{i}}=\mathrm{v}_{\mathrm{i}} \mathrm{v}_{\mathrm{i}+1} \mid 1 \leq \mathrm{i}\right.$ $\leq \mathrm{n}-1\}$ be the edge set of the path $\mathrm{P}_{\mathrm{n}}$. Define a 1-1 function $\mathrm{f}: \mathrm{V} \rightarrow \mathrm{N}$ such that

$$
f\left(v_{i}\right)=2^{\frac{i+1}{2}}
$$

$\mathrm{f}\left(\mathrm{v}_{\mathrm{i}+1}\right)=\mathrm{p} 2^{\frac{\mathrm{n}-\mathrm{i}+2}{2}}$ for $\mathrm{i}=1,3, \ldots$ where $\mathrm{p}<10, \mathrm{p} \neq 2$ is a prime number and an induced edge function $\mathrm{f}^{*}: \mathrm{E} \rightarrow \mathrm{N}$ such that $\mathrm{f}^{*}\left(e_{i}\right)=\mathrm{f}^{*}\left(\mathrm{v}_{\mathrm{i}} \mathrm{v}_{\mathrm{i}+1}\right)=\mathrm{f}\left(\mathrm{v}_{\mathrm{i}}\right) \mathrm{f}\left(\mathrm{v}_{\mathrm{i}+1}\right)$ for $1 \leq \mathrm{i} \leq \mathrm{n}-1$.

Now $f^{*}\left(e_{i}\right)=f^{*}\left(v_{i} v_{i+1}\right)=f\left(v_{i}\right) f\left(v_{i+1}\right)=2^{\frac{i+1}{2}} p 2^{\frac{n-i+2}{2}}=p 2^{\frac{n+3}{2}}$ for $i=1$, $3, \quad 5, \ldots$ is a Zumkeller number and constant for $\mathrm{i}=1,3,5, \ldots$ and $\mathrm{f}^{*}\left(\mathrm{e}_{\mathrm{i}+1}\right)=\mathrm{f}^{*}\left(\mathrm{v}_{\mathrm{i}+1} \mathrm{v}_{\mathrm{i}+2}\right)=\mathrm{f}\left(\mathrm{v}_{\mathrm{i}+1}\right) \mathrm{f}\left(\mathrm{v}_{\mathrm{i}+2}\right)=$ $\mathrm{p} 2^{\frac{\mathrm{n}-\mathrm{i}+2}{2}} 2^{\frac{\mathrm{i}+3}{2}}=\mathrm{p} 2^{\frac{\mathrm{n}+5}{2}}$ is also a Zumkeller number and is constant for $\mathrm{i}=1,3,5, \ldots$. That is, only 2 -Zumkellernumbers are used to label all the edges of the path. Hence the path $P_{n}$ having odd number of vertices admits a 2-Zumkeller labeling.

\section{Lemma 3.3.}

The path $P_{n}$ with $n$ vertices is a 2-Zumkeller graph where $n \equiv 0$ $(\bmod 2)$ and $n \geq 4$.

\section{Proof.}

Let $\mathrm{V}=\left\{\mathrm{v}_{\mathrm{i}} \mid 1 \leq \mathrm{i} \leq \mathrm{n}\right\}$ and $\mathrm{E}=\left\{\mathrm{e}_{\mathrm{i}}=\mathrm{v}_{\mathrm{i}} \mathrm{v}_{\mathrm{i}+1} \mid 1 \leq \mathrm{i} \leq \mathrm{n}-1\right\}$ be the vertex set and edge set of $P_{n}$ respectively. Define a 1-1 function $\mathrm{f}: \mathrm{V} \rightarrow \mathrm{N}$ such that

$$
f\left(v_{i}\right)=2^{\frac{i+1}{2}}
$$

$\mathrm{f}\left(\mathrm{v}_{\mathrm{i}+1}\right)=\mathrm{p} 2^{\frac{\mathrm{n}-\mathrm{i}+\mathrm{l}}{2}}$ for $\mathrm{i}=1,3, \ldots$ where $\mathrm{p}<10, \mathrm{p} \neq 2$ is a prime number and an induced function $\mathrm{f}^{*}: \mathrm{E} \rightarrow \mathrm{N}$ such that $f^{*}\left(e_{i}\right)=f^{*}\left(v_{i} v_{i+1}\right)=f\left(v_{i}\right) f\left(v_{i+1}\right)$ for $1 \leq i \leq n-1$.

Now $f^{*}\left(e_{i}\right)=f^{*}\left(v_{i} v_{i+1}\right)=f\left(v_{i}\right) f\left(v_{i+1}\right)=2^{\frac{i+1}{2}} p 2^{\frac{n-i+1}{2}}=p 2^{\frac{n+2}{2}}$ for $i=1$, $3,5, \ldots$ is a Zumkeller number and constant for $i=1,3,5, \ldots$ and $f^{*}\left(e_{i+1}\right)=f^{*}\left(v_{i+1} v_{i+2}\right)=f\left(v_{i+1}\right) f\left(v_{i+2}\right)=p 2^{\frac{n-i+1}{2}} 2^{\frac{i+3}{2}}=p 2^{\frac{n+4}{2}}$ is also a Zumkeller number and is constant for $\mathrm{i}=1,3,5, \ldots$ That is, only two Zumkeller numbers are used to label all the edges of the path. Hence the path $P_{n}$ having even number of vertices admits a 2-Zumkeller labeling.

\section{Remark 3.1.}

The total number of different Zumkeller numbers used to label the edges of the path is 2 , which is the degree of the non-pendant vertices of the path.

The 2-Zumkeller labeling of the paths $\mathrm{P}_{8}$ and $\mathrm{P}_{9}$ using Lemmas 3.3 and 3.2 are shown in Example 3.1 and Example 3.2.

\section{Example 3.1.}

For $\mathrm{p}=3$ and $\mathrm{n}=8$, the path $\mathrm{P}_{8}$ is a 2-Zumkeller graph.

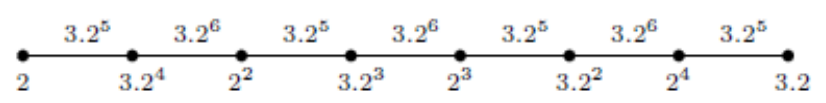

Fig. 2: 2-Zumkeller labeling of $\mathrm{P}_{8}$

Here we use only two Zumkeller numbers viz., $3\left(2^{5}\right)$ and $3\left(2^{6}\right)$ to label the edges of the path $\mathrm{P}_{8}$

\section{Example 3.2.}

For $\mathrm{p}=5$ and $\mathrm{n}=9$, the path $\mathrm{P}_{9}$ is a 2-Zumkeller graph.

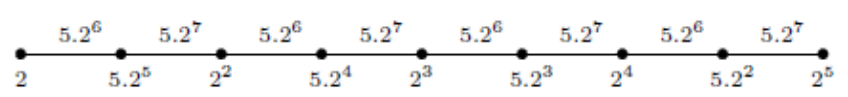

Fig. 3: 2-Zumkeller labeling of $\mathrm{P}_{9}$

\section{Theorem 3.1.}

The path $P_{n}$ with $n$ vertices is a 2-Zumkeller graph where $n \geq 3$.

\section{Theorem 3.2.}

An even cycle $C_{n}$ is a 3-Zumkeller graph.

\section{Proof.}

Let $\mathrm{V}=\left\{\mathrm{v}_{\mathrm{i}} \mid 1 \leq \mathrm{i} \leq \mathrm{n}\right\}$ and $\mathrm{E}=\left\{\mathrm{e}_{\mathrm{i}}=\mathrm{v}_{\mathrm{i}} \mathrm{v}_{\mathrm{i}+1} \mid 1 \leq \mathrm{i} \leq \mathrm{n}-1\right\} \cup\left\{\mathrm{e}_{\mathrm{n}}=\mathrm{v}_{\mathrm{n}}\right.$ $\left.v_{1}\right\}$ be the vertex set and edge set of the even cycle $C_{n}$ respectively. Now the 1-1 function $\mathrm{f}: \mathrm{V} \rightarrow \mathrm{N}$ is defined as in Lemma 3.3, and the edge function $\mathrm{f}^{*}: \mathrm{E} \rightarrow \mathrm{N}$ can be defined as

(i) $\mathrm{f}^{*}\left(\mathrm{e}_{\mathrm{i}}\right)=\mathrm{f}^{*}\left(\mathrm{v}_{\mathrm{i}} \mathrm{v}_{\mathrm{i}+1}\right)=\mathrm{f}\left(\mathrm{v}_{\mathrm{i}}\right) \mathrm{f}\left(\mathrm{v}_{\mathrm{i}+1}\right), 1 \leq \mathrm{i} \leq \mathrm{n}-1$ and

(ii) $f^{*}\left(e_{n}\right)=f^{*}\left(v_{n} v_{1}\right)=f\left(v_{n}\right) f\left(v_{1}\right)$.

In Lemma 3.3, it is proved that $\mathrm{f}^{*}\left(\mathrm{e}_{\mathrm{i}}\right), 1 \leq \mathrm{i} \leq \mathrm{n}-1$ receive only the two Zumkeller numbers $\mathrm{p} 2^{\frac{\mathrm{n}+2}{2}}$ and $\mathrm{p} 2^{\frac{\mathrm{n}+4}{2}}$.

Now $f^{*}\left(e_{n}\right)=f^{*}\left(v_{n} v_{1}\right)=f\left(v_{n}\right) f\left(v_{1}\right)=p 2^{1} 2^{1}=p 2^{2}$ is the third Zumkeller number.

Hence the even cycle $C_{n}$ is a 3-Zumkellergraph.

\section{Corollary 3.1.}

The odd cycle $C_{n}, n \geq 5$ is not a 3-Zumkeller graph.

\section{Remark 3.2.}

The odd cycle $\mathrm{C}_{3}$ is a 3-Zumkeller graph.

\section{Example 3.3.}

The even cycle $\mathrm{C}_{6}$ is a 3-Zumkeller graph for $\mathrm{p}=3$.

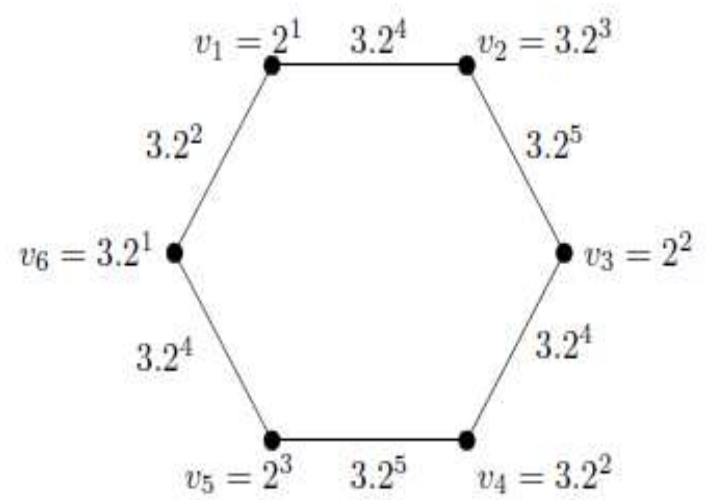

Fig. 4: 3-Zumkeller labeling of $\mathrm{C}_{6}$

Here three distinct Zumkeller numbers viz., $3\left(2^{4}\right), 3\left(2^{5}\right), 3\left(2^{2}\right)$ are used to label the edges of the cycle $\mathrm{C}_{6}$.

\section{Theorem 3.3.}

The comb graph $\mathrm{P}_{\mathrm{n}} \circ \mathrm{K}_{1}$ is a 3-Zumkeller graph. 


\section{Proof.}

Let $\mathrm{V}=\left\{\mathrm{v}_{\mathrm{i}}, \mathrm{u}_{\mathrm{i}} \mid 1 \leq \mathrm{i} \leq \mathrm{n}\right\}$ be the vertex set and $\mathrm{E}=\left\{\mathrm{v}_{\mathrm{i}} \mathrm{v}_{\mathrm{i}+1} \mid 1 \leq \mathrm{i} \leq\right.$ $\mathrm{n}-1\} \cup\left\{\mathrm{v}_{\mathrm{i}} \mathrm{u}_{\mathrm{i}} \mid 1 \leq \mathrm{i} \leq \mathrm{n}\right\}$ be the edge set of the comb graph. Define an injective function $\mathrm{f}: \mathrm{V} \rightarrow \mathrm{N}$ such that

$\mathrm{f}\left(\mathrm{v}_{\mathrm{i}}\right)=2^{\mathrm{i}}$

$\mathrm{f}\left(\mathrm{v}_{\mathrm{i}+1}\right)=\mathrm{p} 2^{\mathrm{n}-\mathrm{i}}$

$\mathrm{f}\left(\mathrm{u}_{\mathrm{i}}\right)=\mathrm{p} 2^{\mathrm{n}-\mathrm{i}+1}$

$\mathrm{f}\left(\mathrm{u}_{\mathrm{i}+1}\right)=2^{\mathrm{i}+1}$; for $\mathrm{i}=1,3, \ldots$ and $\mathrm{p}<10$ and $\mathrm{p}$ is a prime number not equal to 2 .

and an edge function $\mathrm{f}^{*}: \mathrm{E} \rightarrow \mathrm{N}$ such that $\mathrm{f}^{*}(\mathrm{uv})=\mathrm{f}(\mathrm{u}) \mathrm{f}(\mathrm{v})$.

The edge labels of the comb graph are calculated as follows.

(i) $\mathrm{f}^{*}\left(v_{i} v_{i+1}\right)=f\left(v_{i}\right) f\left(v_{i+1}\right)=2^{i} p 2^{n-i}=p 2^{n}$ which is a Zumkeller number and is a constant,

(ii) $\mathrm{f}^{*}\left(\mathrm{v}_{\mathrm{i}} \mathrm{u}_{\mathrm{i}}\right)=\mathrm{f}\left(\mathrm{v}_{\mathrm{i}}\right) \mathrm{f}\left(\mathrm{u}_{\mathrm{i}}\right)=2^{\mathrm{i}} \mathrm{p} 2^{\mathrm{n}-\mathrm{i}+1}=\mathrm{p} 2^{\mathrm{n}+1}$ which is also a

Zumkeller number and is a constant and

(iii) $\left.\mathrm{f}^{*}\left(\mathrm{v}_{\mathrm{i}+1} \mathrm{v}_{\mathrm{i}+2}\right)=\mathrm{f}\left(\mathrm{v}_{\mathrm{i}+1}\right\}\right) \mathrm{f}\left(\mathrm{v}_{\mathrm{i}+2}\right)=\mathrm{p} 2^{\mathrm{n}-\mathrm{i}} 2^{\mathrm{i}+2}=\mathrm{p} 2^{\mathrm{n}+2}$ is also a

Zumkeller number and is a constant.

That is, the edges of the comb graph take exactly 3 Zumkeller numbers.

Hence the comb graph $\mathrm{P}_{\mathrm{n}} \circ \mathrm{K}_{1}$ is a 3-Zumkeller graph.

\section{Example 3.4.}

The 3-Zumkeller labeling pattern of $\mathrm{P}_{6} \circ \mathrm{K}_{1}$ is shown in the Figure 5.

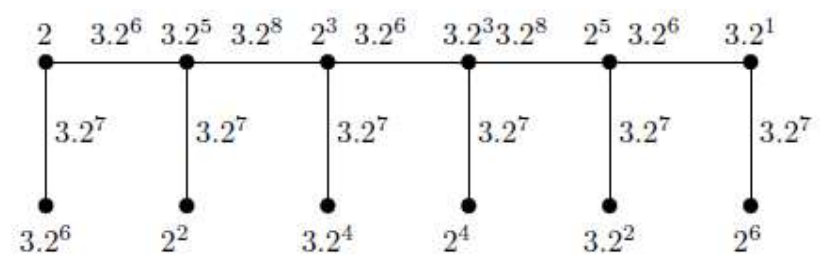

Fig. 5: 3-Zumkeller labeling of a comb graph

\section{Remark 3.3.}

The total number of different Zumkeller numbers used to label the edges of the comb graph is 3 , which is the greatest degree of the non-pendant vertices of the graph.

\section{Corollary 3.2.}

For all $\mathrm{n}$, the ladder graph $\mathrm{L}_{\mathrm{n}}$ admits a 3-Zumkeller labeling.

\section{Proof.}

By joining the vertices $u_{i}$ and $u_{i+1}$ of the comb graph $P_{n} \circ K_{1}$ in the Theorem 3.3, we get the ladder graph $\mathrm{L}_{\mathrm{n}}$.

We follow the same labeling pattern as in the Theorem 3.3.

For the edge $u_{i} u_{i+1}$, we define the edge function $f^{*}$ as

$\mathrm{f}^{*}\left(\mathrm{u}_{\mathrm{i}} \mathrm{u}_{\mathrm{i}+1}\right)=\mathrm{f}\left(\mathrm{u}_{\mathrm{i}}\right) \mathrm{f}\left(\mathrm{u}_{\mathrm{i}+1}\right)=\mathrm{p} 2^{\mathrm{n}-\mathrm{i}+1} 2^{\mathrm{i}+1}=\mathrm{p} 2^{\mathrm{n}+2}$ is a Zumkeller number.

Similarly, for the edge $u_{i+1} u_{i+2}$ we have

$f^{*}\left(u_{i+1} u_{i+2}\right)=f\left(u_{i+1}\right) f\left(u_{i+2}\right)=2^{i+1} p 2^{n-(i+2)+1}=2^{i+1} p 2^{n-i-1}=p 2^{n}$ which is also a Zumkeller number.

Hence the ladder graph $L_{n}$ is a 3-Zumkeller graph.

\section{Example 3.5.}

The 3-Zumkeller labeling of a ladder graph $\mathrm{L}_{5}$ using the above corollary is shown in Figure 6.

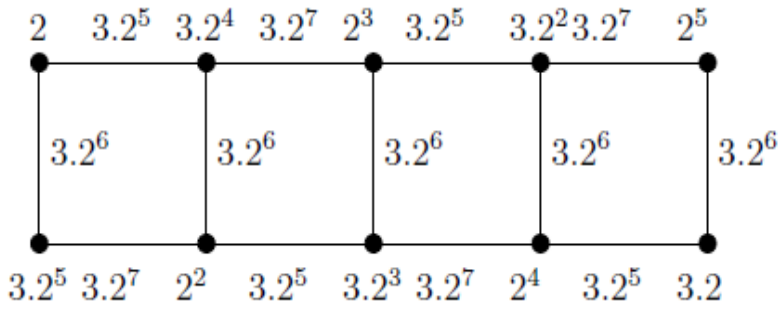

Fig. 6: 3-Zumkeller labeling of the ladder graph $\mathrm{L}_{5}$

\section{Remark 3.4.}

In the 3-Zumkeller ladder graph, 3 represents the greatest degree of the vertices of the graph.

Theorem 3.4.

The square grid $\mathrm{P}_{\mathrm{n}} \times \mathrm{P}_{\mathrm{n}}$ for $\mathrm{n} \equiv 0(\bmod 2)$ admits $(\mathrm{n}+1)$ a Zumkeller labeling.

\section{Proof.}

Let $V=\left\{v_{i j} ; 1 \leq \mathrm{i} \leq \mathrm{n}, 1 \leq \mathrm{j} \leq \mathrm{n}\right\}$ be the vertex set and $\mathrm{E}=\left\{\mathrm{v}_{\mathrm{ij}}\right.$ $\left.\mathrm{v}_{\mathrm{ij}+1}, \mathrm{v}_{\mathrm{ij}} \mathrm{v}_{\mathrm{i}+1 \mathrm{j}}, 1 \leq \mathrm{i} \leq \mathrm{n}-1,1 \leq \mathrm{j} \leq \mathrm{n}-1\right\}$ be the edge set.

Define a 1-1 function $\mathrm{f}: \mathrm{V} \rightarrow \mathrm{N}$ such that

For $\mathrm{i} \equiv 1(\bmod 2)$

$\mathrm{f}\left(\mathrm{v}_{11}\right)=2$

$\mathrm{f}\left(\mathrm{v}_{\mathrm{n}-\mathrm{kn}}\right)= \begin{cases}3.2 & \text { for } \mathrm{k}=1 \\ 2^{\mathrm{k}+1} \mathrm{f}\left(\mathrm{v}_{\mathrm{n}-(\mathrm{k}-2) \mathrm{n}}\right) & \text { for } \mathrm{k} \equiv 1(\bmod 2) \text { and } 3 \leq \mathrm{k} \leq \mathrm{n}+1\end{cases}$

$f\left(v_{1 j}\right)= \begin{cases}2^{j} f\left(v_{1 j+2}\right) & \text { for } j \equiv 0(\bmod 2) \\ 2^{j-2} f\left(v_{1 j-2}\right) & \text { for } j \equiv 1(\bmod 2)\end{cases}$

$f\left(v_{i j}\right)= \begin{cases}2 f\left(v_{i-1 j+1}\right) & \text { for } j+j \equiv 0(\bmod 2) \\ \frac{f\left(v_{i-1 j+1}\right)}{2} & \text { for } i+j \equiv 1(\bmod 2)\end{cases}$

For $\mathrm{i} \equiv 0(\bmod 2)$

$\mathrm{f}\left(\mathrm{v}_{2 \mathrm{n}}\right)=2^{\mathrm{n}-2} \mathrm{f}\left(\mathrm{v}_{2} \mathrm{n}-2\right)$ for $\mathrm{n} \geq 4$

$\left.\mathrm{f}\left(\mathrm{v}_{\mathrm{in}}\right\}\right)=2^{\mathrm{n}-\mathrm{i}+1} \mathrm{f}\left(\mathrm{v}_{\mathrm{i} \mathrm{n}-2}\right)$ for $\mathrm{n} \geq 4, \mathrm{i} \geq 4$

$\mathrm{f}\left(\mathrm{v}_{\mathrm{nn}}\right)=2^{\mathrm{n}^{2} / 2}$

Define an induced edge function

$\mathrm{f}^{*}: \mathrm{E} \rightarrow\left\{3.2^{\frac{\mathrm{n}^{2}}{2}-\mathrm{k}} ; \mathrm{k} \in\left[-\frac{\mathrm{n}}{2}, \frac{\mathrm{n}}{2}\right]\right\}$

Now, the numbers of the form $3.2^{\frac{n^{2}}{2}-k}$ for $k \in N$ are Zumkeller numbers. For completeness of the theorem, it is enough to prove that the number of Zumkeller numbers used to label the edges is $\mathrm{n}+1$. Now the total number of edges in $\mathrm{P}_{\mathrm{n}} \times \mathrm{P}_{\mathrm{n}}$ square grid is $2 n(n-1)$. Out of these edges,

(i) $2 n-3$ edges receive $3.2^{\frac{n^{2}}{2}+\frac{n}{2}}$ for $n \geq 4$

(ii) $2 \mathrm{n}-3$ edges receive $3.2^{\frac{\mathrm{n}^{2}}{2}-\frac{\mathrm{n}}{2}}$ for $\mathrm{n} \geq 4$

(iii) $4 \mathrm{~m}-10$ edges receive $3.2^{\frac{\mathrm{n}^{2}}{2} \pm\left(\frac{\mathrm{m}-2}{2}\right)}$ for $\mathrm{n} \geq \mathrm{m}$ and $\mathrm{m}$ is even, $\mathrm{m}>2$ and

(iv) the edges $e_{12,1}$ and $e_{n, n-1 n}$ receive $3 \cdot 2^{\frac{n^{2}}{2}}$.

Hence the proof.

The 5-Zumkeller labeling of the square grid $\mathrm{P}_{4} \times \mathrm{P}_{4}$ is given in the following example.

\section{Example 3.6.}

The total number of edges in this graph is $2 \times 4 \times 3=24$. From the vertex function defined in the above theorem, the range of the edge set is $\left\{3.2^{6}, 3.2^{7}, 3.2^{8}, 3.2^{9}, 3.2^{10}\right\}$. Out of these $2 n-3=2 \times 4$ $-3=5$ edges receive $3.2^{8 \pm 2}$. That is, 5 edges receive $3.2^{10}$ and 5 edges receive $3.2^{6}$. For $\mathrm{m}=4,4 \mathrm{~m}-10=4 \times 4-10=6$ edges receive $3.2^{\frac{4^{2}}{2} \pm\left(\frac{4-2}{2}\right)}=3.2^{8 \pm 1}$.

That is, 6 edges receive the Zumkeller number $3.2^{7}, 6$ edges receive the Zumkeller number $3.2^{9}$, the edges $\mathrm{e}_{12,1}$ and $\mathrm{e}_{4,34}$ receive 
the Zumkeller number $3.2^{8}$. In total $5+5+6+6+2=24$ edges of $\mathrm{P}_{4} \times \mathrm{P}_{4}$ receive only 5-Zumkellernumbers.

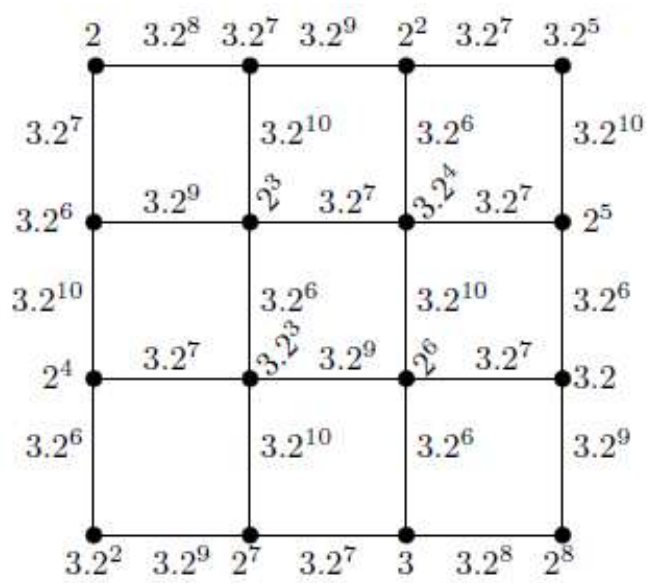

Fig. 7: 5-Zumkeller labeling of the square grid $\mathrm{P} 4 \times \mathrm{P} 4$

\section{Conclusion}

In this article, we proved that the graphs viz., paths, cycles, comb graphs, ladder graphs and square grid $\mathrm{P}_{\mathrm{n}} \times \mathrm{P}_{\mathrm{n}}$ are k-Zumkeller graphs.

\section{References}

[1] Balamurugan BJ, Thirusangu K, Thomas DG (2013), Strongly multiplicative Zumkeller labeling of graphs. International Conference on Information and Mathematical Sciences, Elsevier, 349-354.

[2] Balamurugan BJ, Thirusangu K, Thomas DG (2014), Zumkeller labeling of some cycle related graphs. Proceedings of International Conference on Mathematical Sciences (ICMS - 2014), Elsevier, 549-553.

[3] Balamurugan BJ, Thirusangu K, Thomas DG (2015), Zumkeller labeling algorithms for complete bipartite graphs and wheel graphs. Advances in Intelligent Systems and Computing, Springer 324, 405-413.

[4] Balamurugan BJ, Thirusangu K, Thomas DG (2015), Algorithms for Zumkeller labeling of full binary trees and square grids. Advances in Intelligent Systems and Computing, Springer 325, 183-192.

[5] Balamurugan BJ, Thirusangu K, Thomas DG (2015), k-Zumkeller labeling for twig graphs. Electronic Notes in Discrete Mathematics, Elsevier 48, 119-126.

[6] Bloom GS, Golomb SW (1977), Applications of numbered undirected graphs. IEEE, 165 of 4, 526-570.

[7] Gallian JA (2016), A dynamic survey of graph labeling. Electronic Journal of Combinatorics 17 (DS6).

[8] Harary F, Graph theory. Addison-Wesley, Reading Mass, (1972).

[9] Murali BJ, Thirusangu K, Balamurugan BJ (2017), Zumkeller cordial labeling of cycle related graphs. International Journal of Pure and Applied Mathematics 116(3), 617-627.

[10] Richard Johnsonbaugh, Discrete Mathematics. Pearson Education, Asia, (2001).

[11] Rosa A (1966), On certain valuations of the vertices of a graph. In N.B. Gordan and Dunad, editors, Theory of graphs. International Symposium, Paris, 349-359.

[12] Srinivasan AK (1948), Practical numbers. Current Science 17, 179-180.

[13] YuejianPeng, BhaskaraRao KPS (2013), On Zumkeller numbers. Journal of Number Theory 133(4), 1135-1155. 